
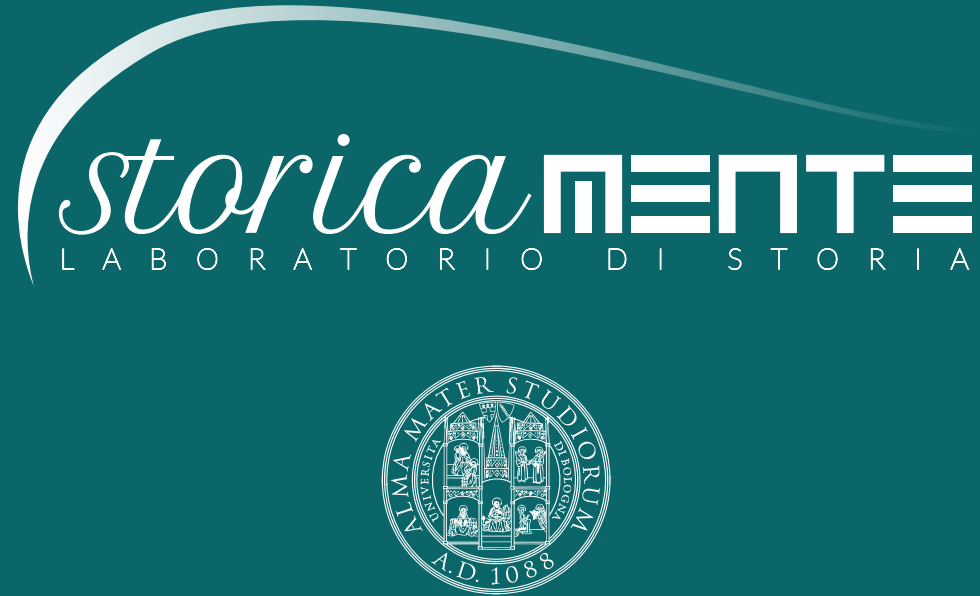

ALMA MATER STUDIORUM

Università di Bologna

Dipartimento di Storia Culture Civiltà

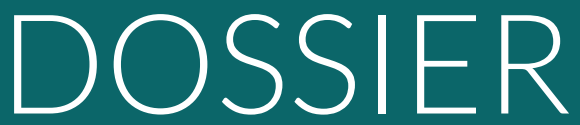

LA POLITICA OLTRE L'IMPERATORE: STRUTTURE, STRATEGIE ED EVOLUZIONE DEI POTERI DIETRO IL TRONO A ROMA E A BISANZIO 


\section{STORICAMENTE.ORG}

\section{Laboratorio di Storia}

Simone Ciambelli

Il patronato interessato dei senatori presso le associazioni professionali di Ostia antica

Numero 14 - 2018

ISSN: 1825-411X

Art. 50

pp. 1-27

DOI: $10.12977 /$ stor740

Editore: BraDypUS

Data di pubblicazione: 27/05/2019

Dossier: La politica oltre l'imperatore: strutture, strategie ed evoluzione dei poteri dietro il trono a Roma e a Bisanzio 


\title{
Il patronato interessato dei senatori presso le associazioni professionali di Ostia antica
}

\author{
SIMONE CIAMBELLI
}

Univ., Dipartimento Storia Culture e Civiltà

Senators need the support of plebs media like the emperor needs the senators' one. This paper will focus on the patronage of professional associations in Ostia ruled by senators. The analysis of this particular relationship allows us to scrutinize one of the means used by the members of the imperial elite to establish solid relationships with the merchant class, necessary in order to perpetuate their socio-economic stability. Furthermore, this research underlines the importance of the associative milieu in Ostia, strong enough to attract the interest of senators.

\section{Introduzione alla situazione ostiense}

Così come il potere dei principes, proveniente in linea teorica dal popolus, era soggetto in quasi tutta l'epoca imperiale agli spostamenti delle "lobby" aristocratiche, così i senatori necessitavano del sostegno della plebs media. Attraverso l'analisi del fenomeno del patronato sulle associazioni professionali ostiensi esercitato dai senatori è possibile individuare uno dei mezzi utilizzati dai membri dell'élite imperiale per instaurare saldi rapporti con il ceto mercatile, necessari al fine di perpetuare la loro stabilità socio-economica. 
Dalle fonti antiche emerge che sin dall'epoca mediorepubblicana la risalita del Tevere era preclusa alle grandi imbarcazioni a causa del continuo insabbiamento della foce'. Numerosi furono gli interventi per cercare di risolvere questo enorme problema che impediva il rapido approvvigionamento di Roma, megalopoli che non poteva più sopravvivere basandosi esclusivamente sul suo contado.

Una prima efficace soluzione fu trovata da Claudio, che nel 42 d.C. intraprese la costruzione di un nuovo porto due chilometri a nord di Ostia (nell'odierno Comune di Fiumicino), ma bisognerà attendere il principato di Nerone e precisamente il 64 d.C. per l'inaugurazione ufficiale del porto, ricordata su diverse serie di sesterzi emanati dalle zecche di Lugdunum e Roma (figura 1). La struttura del porto doveva risultare maestosa: un

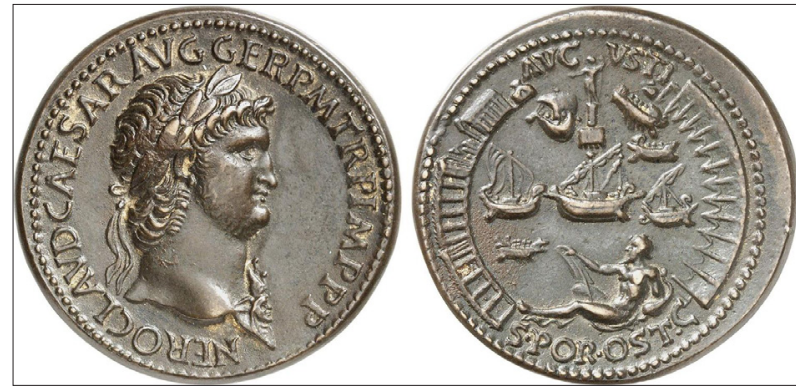

Figura 1. Sesterzio di Nerone, 64 d.C. Al dritto testa laureata dell'imperatore. Al rovescio vista del porto di Ostia: in alto il faro sovrastato da Nettuno; in basso la figura del Tevere regge nella sua mano un delfino; nel mezzo sette navi attorniate dalle strutture portuali (foto: <www.numismatics.org/ocre/>).

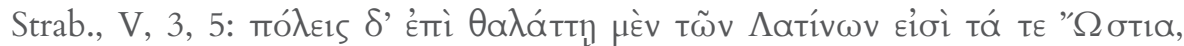

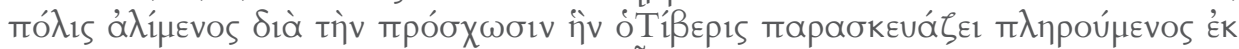

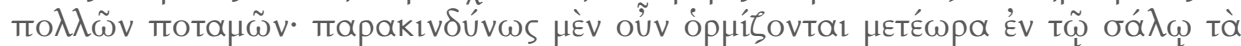

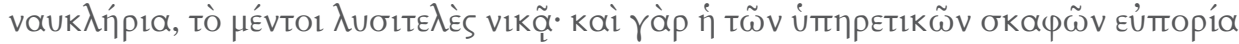

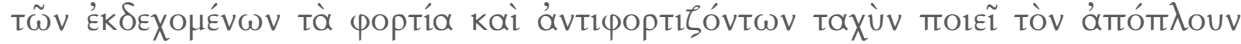

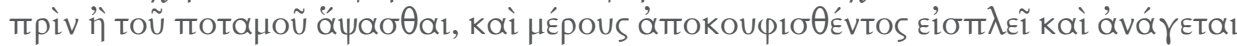

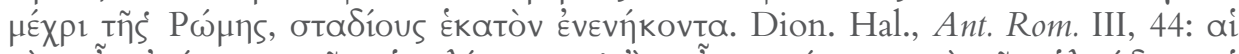

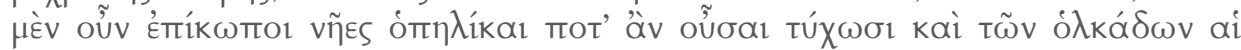

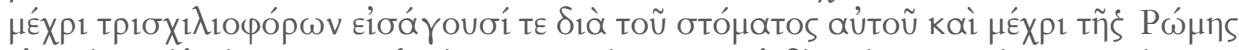

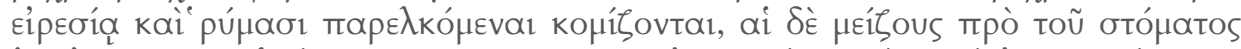

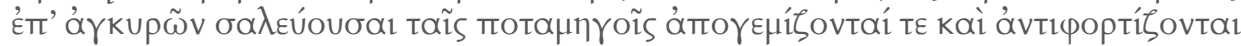

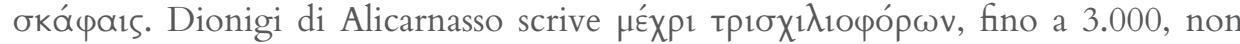
specificando l'unità di misura la quale, tuttavia, normalmente era espressa in anfore, quindi il passo è da leggere: fino a 3000 anfore. 3.000 anfore $=3.000$ talenti $=9.000$ modii $=78$ tonnellate circa [Meiggs 1973, 51]. 
grande bacino della capacità di circa 80 ettari [Chevallier 1986, 120] era delimitato da due lembi di terra che si distaccavano dalla costa a semicerchio senza incontrarsi, e nel loro mezzo si ergeva un faro che ricordava quello di Alessandria². Il porto assunse il nome ufficiale di portus Augusti Ostiensis e divenne il nuovo scalo di Ostia, senza per questo soppiantare il vecchio porto fluviale della città [Meiggs 1973, 85-86]. Nonostante ciò, la tanto ricercata sicurezza non fu raggiunta. Tacito negli Annales scrisse che nel 62 d.C. circa 200 navi alla fonda nel porto furono spazzate via da una violenta onda anomala ${ }^{3}$, probabilmente causata dallo stesso brutale terremoto che provocò ingentissimi danni a Pompei [Zevi 2001, 280].

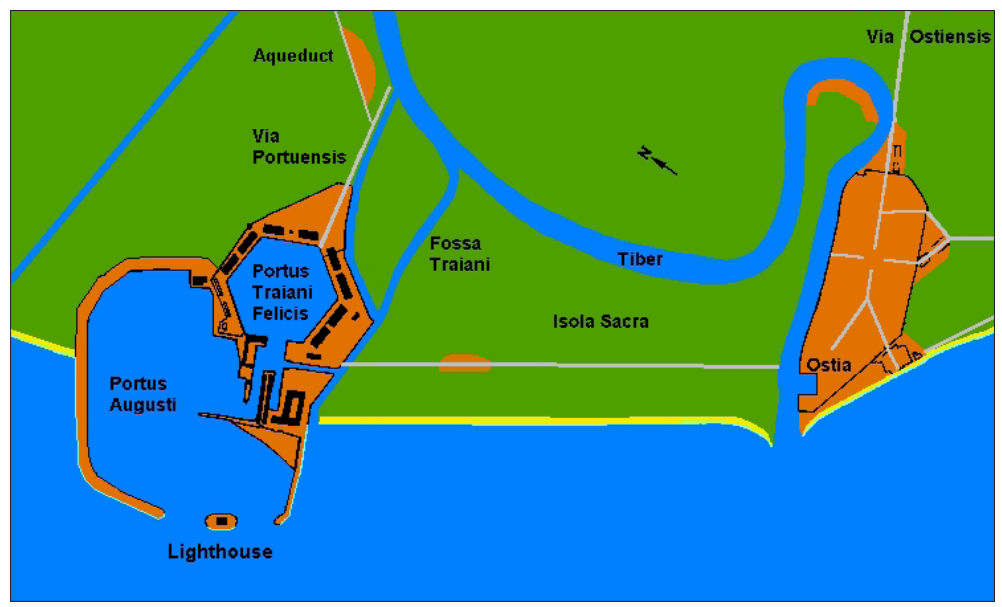

Figura 2. Ostia e i porti imperiali di Portus (foto: <www. ostia-antica. org $>$ )

Svet., Claud., 20, 3: Portum Ostiae extruxit circumducto dextra sinistraque brachio et ad introitum profundo iam solo mole obiecta; quam quo stabilius fundaret, navem ante demersit, qua magnus obeliscus ex Aegypto fuerat advectus, congestisque pilis superposuit altissimam turrem in exemplum Alexandrini Phari, ut ad nocturnos ignes cursum navigia

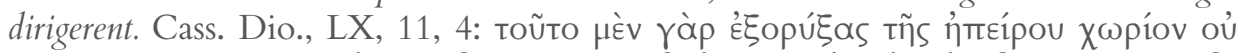

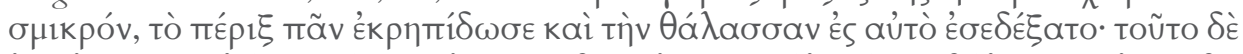

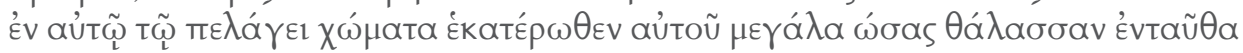

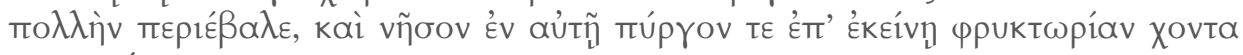
кат єоти́бато.

Tac., Ann., XV,18, 2: cuius pretio nihil additum est, quamvis ducentas ferme navis portu in ipso violentia tempestatis. Il porto nel 62 d.C. era già in funzione, anche se effettivamente fu inaugurato nel 64 d.C. 
La soluzione definitiva fu la costruzione, sotto gli auspici di Traiano, di un bacino interno al porto di Claudio di forma esagonale, forse progettato dall'architetto di fiducia dell'imperatore, Apollodoro di Damasco [Zevi 2001, 280] (figura 2). Tuttavia Ranuccio Bianchi Bandinelli assume una posizione molto cauta nell'attribuire quest'opera all'autore del foro di Traiano: «ipotesi moderne, non dimostrabili, gli hanno attribuito il porto artificiale situato fra Ostia (Porto) e Fiumicino» [Bianchi Bandinelli 1969, 239].

La costruzione del nuovo porto di Traiano, che assunse il nome ufficiale di portus Traiani felicis, prese il via non prima della fine della campagna in Dacia, condotta dall'imperatore in persona e conclusa nel 106 d.C.; verosimilmente fu una parte del bottino di questa guerra a finanziarne il progetto [Meiggs 1973, 489]. L'inaugurazione dello scalo si fa tradizionalmente risalire, attraverso la documentazione numismatica (figura 3), a un periodo compreso tra il 112 d.C. e l'estate del 114 d.C.

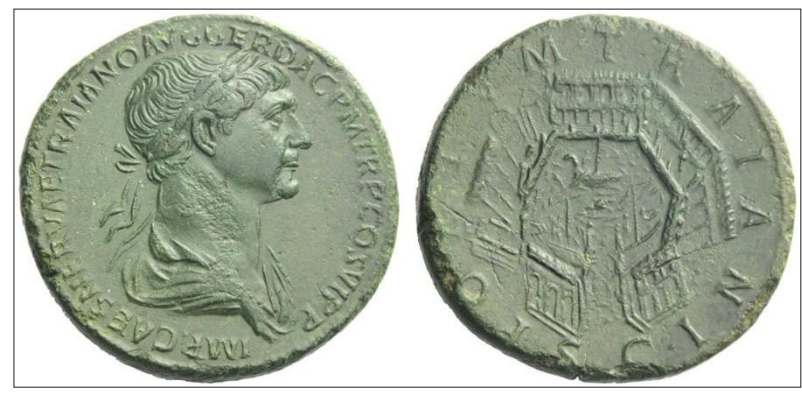

Figura 3. Sesterzio di Traiano, 112-4 d.C. Sul dritto testa di Traiano laureata. Sul verso porto esagonale di Ostia con al centro una nave (foto: <www. numismatics.org/ocre/>).

Tra la fine del primo secolo e gli inizi del terzo il fermento commerciale ed economico causato dalla costruzione dei due porti imperiali influenzò ogni aspetto della vita cittadina ostiense, dall'espansione urbanistica all'esponenziale crescita demografica, favorendo anche la fioritura di numerosi collegia professionali sempre più specializzati e di interesse non solamente locale. 


\section{Nota minima sul fenomeno del patronato}

Partendo dal presupposto che il patronato sia una relazione di dipendenza tra individui e che tali relazioni sono insite in ogni società umana non utopica, è ora opportuno concentrarsi sul ruolo del patronato all'interno della società romana. Richard Saller individuò tre elementi che caratterizzano il patronato: in primo luogo esso è contraddistinto da un reciproco scambio di beni e servizi; in secondo luogo, per distinguere questo scambio dalle transazioni commerciali, deve sussistere un rapporto personale e di una certa durata tra le parti; infine, tale rapporto deve essere asimmetrico, ovvero le due parti devono essere di diversa estrazione sociale e offrire differenti tipi di beni e di servizi in base al ruolo che essi hanno all'interno della relazione [Saller 1982, 1].

Numa Denis Fustel de Coulanges prima (1874) e Matthias Gelzer poi (1912), individuarono quattro differenti categorie di patronato che si svilupparono nella società romana: il patronato nel rapporto clientelare, il rapporto patrono-liberto, il patronato giuridico e il patronato delle collettività, entro il quale rientra il caso specifico del patronato sulle associazioni professionali [Fustel de Coulanges 1900, 205-225; Gelzer 1962, 69].

Per il Waltzing «les collèges imitèrent les villes» [Waltzing 1895, 426] e proprio a imitazione delle comunità cittadine iniziarono anche loro $\mathrm{a}$ fornirsi della figura del patrono. Il patrono dei collegia era di norma una figura "en dehors et au-dessus des fonctionnaires des collèges» [ivi, 425] che diveniva tale attraverso la cooptatio patroni per opera del collegium stesso. La cooptatio avveniva attraverso una votazione nell'assemblea generale di tutto il collegio (collegium universi) all'interno della schola o del tempio in cui i soci si riunivano. Il testo di tale proposta, in caso di risposta affermativa del prescelto, veniva inciso su una tabula patronatus, in genere bronzea, che veniva affissa nella casa del patronus, mentre una copia era destinata a essere conservata nel tabularium del collegium [ivi, 429]. 
Una volta che il patrono aveva accettato gli onori offertigli dall'associazione, si stabiliva tra lui e i collegiati lo stesso legame che è possibile riscontrare nel rapporto tra patrono e cliente [ivi, 430]ث. Il collegium cercava in tutti i modi di accattivarsi l'attenzione del patrono e della sua famiglia attraverso la concessione di numerosi onori: il nome dei patroni era sempre posto in testa negli alba dei collegia e di norma scritto in caratteri di maggiori proporzioni rispetto a quelli usati per incidere i nomi dei quinquennales; egli inoltre poteva assistere all'assemblea dell'associazione, dove, pur non potendo votare, poteva pronunciare discorsi che avevano un enorme peso nella votazione successiva ${ }^{5}$. Il col-

4 Questa è un'interpretazione del Waltzing, che sembra del resto essere confermata da un'iscrizione rinvenuta su una base di statua a Pozzuoli che riporta, CIL X, $1696=$ ILS 1226 = [Waltzing 1899, 436-437] nr. 1672 = EDR 108157 (Giuseppe Camodeca): Mavorti Iun(iores) / Q(uinto) Flavio Maesio / Cornelio Egnatio / Severo Lolliano, / c(larissimo) p(uero), q(uaestori) k(andidato), / Decatrenses cli/entes eius patrono / praestantissimo / posuerunt. Quintus Flavius Maesius Cornelius Egnatius Severus Lollianus è descritto come patrono dei Decatrenses, attestati in un'altra base di statua, posta in onore del padre di lui, come colligium Decatressium, CIL X, $1696=$ ILS 1224c $=$ [Waltzing 1899, 436] nr. 1671: Mavortii // Q(uinto) Flavio Maesio Egnatio Lolliano, c(larissimo) $v$ (iro), I q(uaestori) k(andidato), praetori urbano auguri publico po/puli Romani Quiritium, cons(ulari) albei (!) Ti/beris et cloacarum, cons(ulari) operum publicum, cons(ulari) aquarum, cons(ulari) Camp(aniae), comiti / Flaviali, comiti orientis, I comiti\{s\} primi / ordinis et proconsuli provinciae Africae, / colligeus (!) Decatressium patrono dignis/simo posuerunt. Questo collegium Decatressium, che probabilmente, seguendo l'ipotesi di Giulio Minervini riportata anche dal Waltzing, era composto da un gruppo di mercanti prove-

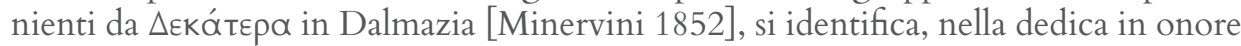
di Mavortius iunior, come u gruppo di clientes eius.

5 CIL VI, 1690 = ILS 1240 = LSA 1396 = EDR 076346 (Antonella Ferraro): Populonii. / L L(ucio) Aradio Val(erio) Proculo, v(iro) c(larissimo), I auguri, / pontifici maiori, I quindecemviro sacris faciundis, I pontifici Flaviali, I praetori tutelari, / legato pro praetore provinciae Numidiae, I peraequatori census provinciae Calleciae, I praesidi provinciae Byzacenae, / consulari provinciae Europae et Thraciae, / consulari provinciae Siciliae, / comiti ordinis secundi, / comiti ordinis primi, / proconsuli provinciae Africae vice / sacra iudicanti, eidemq(ue) iudicio sacro / per provincias Proconsularem et Numidiam Byzacium ac Tripolim, / itemque Mauretaniam Sitifensem et Caesariensem, I perfuncto officio praefecturae praetorio, / comiti iterum ordinis primi intra palatium, / praefecto urbi vice sacra iterum iudicanti, / consuli ordinario, / huic corpus suariorum et confecturariorum, / auctoribus patronis, ex affectu eidem iure debito / statuam patrono digno ponendam censuit. Questa base di statua, dedicata a L. Aradius Valerius Proculus, console nel 340 d.C., cfr. PLRE I, 
legium al momento della cooptatio, oppure in risposta a un favore o a una donazione ottenuta, poteva erigere una statua al patrono, che a volte poteva essere collocata in un luogo pubblico, previa autorizzazione dei decurioni, e quindi avere grandissima visibilità

D'altro canto il notabile si impegnava a pagare il patronalis honor, altra usanza che andava a ricalcare ciò che avveniva a livello municipale con il pagamento della summa honoraria da parte dei magistrati neoeletti, oppure dei personaggi che divenivano decurioni senza essere stati eletti magistrati, per supplire i vuoti che si erano aperti nel consiglio municipale . Numerose sono anche le attestazioni di fondazioni che i patroni fecero in favore dei collegia, soprattutto per celebrare ogni anno, e potenzialmente in perpetuo, il loro compleanno ${ }^{8}$.

pp. 747-749, Proculus 11, del quale viene riportata la brillante carriera, era stata votata grazie all'auctoritas patronis, di cui faceva certamente parte anche il nostro onorato. Ciò sottintende una partecipazione, seppur esterna, dei patroni nelle attività dell'assemblea, almeno per questo collegium.

6 La presenza della formula epigrafica $L$ (ocus) $D$ (atus) $D$ (ecretum) $D$ (ecorionum) scioglie qualsiasi dubbio sulla collocazione in un luogo pubblico della statua dell'onorato.

7 Isidoro di Siviglia così scrive, Isid., Etym., IX, 423: Non est decurio qui summam non intulit. In una delle numerose lettere scritte da Plinio il Giovane a Traiano, il governatore del Ponto e della Bitinia fa presente all'imperatore che coloro che divenivano decurioni per adlectio da parte dei quinquennales non erano tenuti a pagare la summa honoraria, tuttavia è usanza che paghino 1.000 o 2.000 denari. Plin., Ep., X, 112 (113): C. PLINIVS TRAIANO IMPERATORI. Lex Pompeia, domine, qua Bithyni et Pontici utuntur, eos, qui in bulen a censoribus leguntur, dare pecuniam non iubet; sed ii, quos indulgentia tua quibusdam ciuitatibus super legitimum numerum adicere permisit, et singula milia denariorum et bina intulerunt. Anicius deinde Maximus proconsul eos etiam, qui a censoribus legerentur, dumtaxat in paucissimis cinitatibus aliud aliis iussit inferre. Superest ergo, ut ipse dispicias, an in omnibus ciuitatibus certum aliquid omnes, qui deinde buleutae legentur, debeant pro introitu dare. Nam, quod in perpetuum mansurum est, a te constitui decet, cuius factis dictisque debetur aeternitas.

${ }^{8}$ Ad esempio Titus Furius Primigenius, patrono del collegium fabrum tignuariorum di Tolentinum, aveva donato in fondazione all'associazione 10.000 sesterzi affinché ogni anno, nel giorno del suo die natalis, il 21 luglio, si tenesse un banchetto in suo onore. CIL IX, 5568 = ILS $7256=$ [Cristofori 2004, 456] = EDR 015127 (Simona Antolini): Exs(enatus) c(onsulto) / schola Aug(usta) colleg(ii) fabror(um) / tignuar(iorum) impendi(i)s ipsorum ab in/choato exstructa, solo dato ab (!) T(ito) Fu/rio Primigenio, qui et 
Il patrono, oltre che con donazioni materiali, poteva intercedere presso l'organizzazione municipale, o addirittura imperiale, per ottenere servizi o esclusive in favore dei suoi protetti. Egli, inoltre, aveva il compito di prendere le difese legali del collegio, qualora si fosse presentata l'occasione. In tal caso il patronus stesso assumeva il titolo di defensor collegii che "exprimait mieux encore le rôle de protecteur, que le patron, lui aussi, devait assumer en toutes circonstances» [Waltzing 1895, 439]. Alessandro Severo intervenne in modo incisivo nell'organizzazione delle associazioni e impose a ogni collegio di scegliere un defensor che potesse sostenere i processi della comunità, e, a causa del loro rango sociale, furono proprio i patroni ad assumere nella maggioranza dei casi questa funzione [Waltzing 1895, 418]. Tale imposizione dall'alto non faceva altro che confermare una prassi che già da tempo si poteva riscontrare presso varie associazioni.

Lo scambio di onori e di beni materiali tra i collegia e i loro patroni non si esauriva, dunque, nel momento della cooptatio patroni, ma era continuo nel tempo. I collegiati conoscevano molto bene l'arte di stimolare gesti evergetici da parte del loro patrono, non a caso la maggior parte delle iscrizioni riguardanti i patroni sono incise su basi di statue erette in loro onore. I collegia non perdevano neanche un'occasione per decretare in favore del loro protettore "honneurs intéressés» [Waltzing 1895, 432].

Infine, a mio avviso, uno degli aspetti più rilevanti di questa particolare relazione di patronato è che i notabili, soprattutto locali, accrescevano il loro prestigio e riconfermavano la loro posizione all'interno della società attraverso l'esibizione di tabulae patronatus nell'atrium delle loro

dedic(atione) eius HS X(milia) n(ummum) ded(it), I ex cuius summ(ae) redit(u) omnib(us) annis XII K(alendas) August(as) / die natalis sui epulentur. Per approfondimenti si veda Cristofori 2004 [455-465], con bibliografia annessa.

9 SHA, Alex. Sev., 23, 2: corpora omnium constituit vinariorum, lupinariorum, caligariorum et omnino omnium artium hisque ex sese defensores dedit et iussit, qui ad quos iudices pertinerent. 
domus, grazie a iscrizioni riguardanti le loro gesta poste in luoghi pubblici e privati e, soprattutto, grazie alla presenza di statue erette in loro onore, o in onore di personaggi della loro famiglia, in luoghi pubblici che potevano essere centrali, come il forum cittadino o, nel caso di Ostia, il Piazzale delle Corporazioni.

\section{I patroni dei collegia di rango senatorio}

Lo sviluppo del fenomeno del patronato sulle associazioni professionali a Ostia, come quasi tutti gli aspetti della vita di questa colonia, segue diverse direttrici rispetto alla maggior parte dei centri abitati dell'impero. Infatti, se prendiamo in considerazione solo i dati certi e dunque escludiamo la grossa fetta occupata dai personaggi di rango ignoto che è del $47 \%$, il rango sociale dei patroni ostiensi risulta essere estremamente eterogeno. Il dato più sorprendente che emerge è l'elevato numero di senatori: su un totale di 90 patroni ben 15, ovvero il 17\%, era di rango senatorio $^{10}$ (grafico 1$)$.

Come si può notare dalla tabella 1 solamente tre associazioni ostiensi ebbero l'onore di avere come patroni esponenti dell'ordine senatorio, esse furono: il corpus lenunculariorum tabulariorum auxiliariorum Ostiensium, l'ordo corporatorum qui pecunia ad templum contulerunt, ovvero il corpus lenunculariorum traiectus Luculli, e il corpus scaphariorum traiectus Rusticeli.

I lenuncularii erano letteralmente coloro che possedevano i lenunculi (diminutivo di lembus), delle piccole barche utilizzate sia la navigazione marina che fluviale ${ }^{\Perp}$. In tutto il mondo romano i lenuncularii sono

\footnotetext{
10 Nella tabella 1 vi è l'elenco di tutti i patroni di rango senatorio di Ostia.

11 Tra i testi letterari che fanno menzione dei lenunculi ricordo Caes., Bell. Civ., II, 43, 3: Horum fuga navium onerariarum magistros incitabat; pauci lenunculi ad officium imperiumque conveniebant. Tac., Ann., XIV, 5: Verum Acerronia, imprudentia dum se Agrippinam esse utque subveniretur matri principis clamitat, contis et remis et quae fors obtulerat
} 


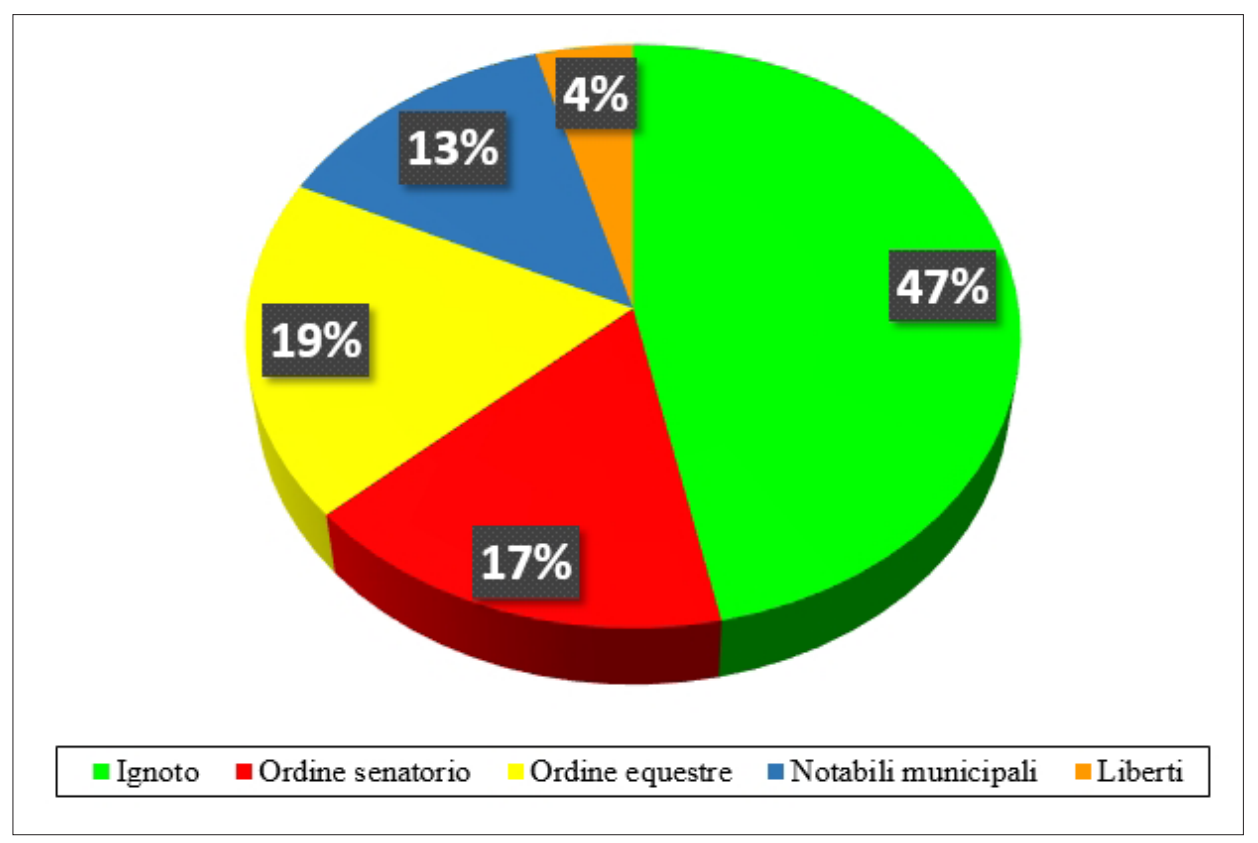

Grafico 1. Il rango sociale dei patroni dei collegia di Ostia.

attestati solamente nelle prospere città commerciali di Ostia e Arelate. Inoltre la loro totale assenza nel materiale epigrafico di Roma ci spinge a considerare l'attività di questi lavoratori esclusivamente in connessione con le strutture portuali poste all'imbocco di due tra i più importanti fiumi dell'occidente romano: il Tevere e il Rodano [Le Gall 1953, 220; Tran 2014, \21]. Dalle iscrizioni ostiensi emergono almeno tre associazioni di lenuncularii: il corpus lenunculariorum tabulariorum auxiliariorum Ostiensium ${ }^{12}$, il corpus lenunculariorum auxiliariorum pleromario-

navalibus telis conficitur: Agrippina silens eoque minus adgnita (unum tamen vulnus umero excepit) nando, deinde occursu lenunculorum Lucrinum in lacum vecta villae suae infertur. Amm. Marc. XIV, 2, 10: Nam sole orto magnitudine angusti gurgitis sed profundi a transitu arcebantur et dum piscatorios quaerunt lenunculos vel innare temere contextis cratibus parant, effusae legiones, quae hiemabant tunc apud Siden, isdem impetu occurrere veloci.

12 CIL XIV, 250= ILS 6174: M(anio) Acilio Glabrione, M(arco) Valerio Homulo co(n) s(ulibus). / Ordo corporatorum lenuncularior(um) / tabulariorum auxiliares (!) Ostiens(ium). // Patroni: / M(arcus) Sedatius C(ai) fil(ius) Severianus, / T(itus) Prifernius Sex(ti) f(ilius) Paetus Rosianus Geminus, I M(arcus) Sedatius M(arci) f(ilius) Severus Iulius Reginus, I 
rum Ostiensium ${ }^{13}$ e il corpus lenunculariorum traiectus Luculli ${ }^{14}$. Per quanto concerne i primi due corpora, la critica moderna ha tentato di chiarire il significato dei termini auxiliarius, comune a entrambi, tabularius e pleromarius per comprendere la funzione di questi collegiati. Il termine auxiliarius significa probabilmente che queste corporazioni svolgevano azioni di aiuto nei confronti dei naviganti della regione ostiense, anche se non sappiamo esattamente di che tipo di aiuto si trattasse [Rougé 1966, 198; Sirks 1991, 274].

Invece, di più difficile interpretazione sono i vocaboli pleromarius e tabularius. Accantonando l'interpretazione di pleromarius ${ }^{15}$, non centrale nell'economia di questo scritto, il termine tabularius può essere interpretato principalmente in due modi. Si potrebbe seguire la traduzione letteraria, ovvero contabile, e dunque si vedrebbe in questi lenuncularii gli operatori portuali incaricati di controllare le carte di bordo e di trainare le navi presso la banchina a loro assegnata [Casson 1965, 35]. Oppure si potrebbe considerare il termine tabularius in stretta connessione

C(aius) Allius C(ai) fil(ius) Fuscianus, // L(ucius) Volusius Maecianus, / L(ucius) Iulius Memor, / M(arcus) Cipius Proclianus, / T(itus) Aurelius Aug(usti) lib(ertus) Strenion, I L(ucius) Marius Germanus. // Quinq(uennalis) perp(etuus): / M(arcus) Cornelius Epagathus. / Quinq(uennalis): / M(arcus) Antistius Helius. / Q(uin)q(uennalis): / M(arcus) Cornelius M(arci)f(ilius) / Secundus. Seguono su tre colonne i nomi di circa 113 membri della plebs.

13 CIL XIV, 252 (fr. a) + p. 614 (fr. b) = ILS 6176 (fr. a) = EDR 143852 (Raffaella Marchesini): Ti(berio) Claudio Severo et C(aio) Aufidio Victorino co(n)s(ulibus). I Ordo corporator(um) lenunculariorum / pleromariorum auxiliarior(um) Ost(i)en[s]ium. I Patr(oni): M(arcus) Cipius Vitalis et T(itus) Antistius Favor. / Q(uin)q(uennales): L(ucius) Veius Adiutor, M(arcus) Cloelius Carus, I P(ublius) Ciarcius Vitalis, M(arcus) Cipius Fortis, / C(aius) Fonteius Fortunatus, Q(uintus) Atilius Maritimus. Seguono i nomi di 16 membri della plebs.

14 CIL XIV, 5380 (fr. a) + Licordari 1987, p. 157, nr. 5 = AE 1987, 197 = EDR 110216 (Raffaella Marchesini): L(ucio) Aelio Commo[do], I Imp(eratoris) Antonini Aug(usti) [Pii, p(atris)] p(atriae), fil(io), / [L(ucius)] Naevius Saecula[ris e]t / [P(ublius) Sul]picius Hera, Augusta[lis] / qui(nque)nnalicius corporis / traiectus Luculli, sua p(ecunia) p(osuerunt).

15 Su pleromarius si vedano Waltzing 1896, 75; Rougé 1966, 198; Sirks 1991, 274; Tran 2014, \$22. 
con tabula, nel senso di codex e dunque classificarlo come sinonimo di codicarius [Waltzing 1896, 76]. Nonostante queste interpretazioni concordo con quanto già affermato da Russell Meiggs: «the functions of the tabularii and pleromarii can only be guessed» [Meiggs 1973, 279]. Si può solamente concludere che i tabularii e i pleromarii, non essendo attestati in alcun modo a Roma, svolgessero le loro attività nei pressi dei porti ostiensi e della foce del Tevere e che queste attività fossero in qualche modo d'aiuto alle navi che giungevano a Ostia.

Sul corpus lenunculariorum traiectus Luculli disponiamo di maggiori informazioni. Traiectus significa passaggio da una parte all'altra del fiume, e ciò qualifica questi lavoratori come coloro che svolgevano il servizio di trasporto da una sponda all'altra del Tevere. Questa attività era essenziale in quanto l'Isola Sacra era raggiungibile via terra solo da nord attraverso un ponte di epoca traianea, mentre per il resto era necessario sfruttare i traghetti.

Probabilmente Lucullus si riferiva al nome del proprietario dei terreni sulle sponde del fiume nei quali vi erano i punti di imbarco dei traghetti [Licordari 1987, 149], oppure stava a designare il nome di colui a cui originariamente era stato appaltato un tratto di banchina fluviale, che a sua volta aveva affidato ai battellieri per l'attracco [Pavolini 1986, 96]. Il corpus lenunculariorum traiectus Luculli era atto sia al trasporto di persone che di merci [Meiggs 1973, 297].

Altro caso ancor più problematico è quello dell'ordo corporatorum qui pecuniam ad ampliandum templum contulerunt. A Ostia sono stati rinvenuti tre alba, completi di patroni, magistrati e collegiati semplici, riportanti la questa intestazione ${ }^{16}$. Questi documenti hanno tutti i requisiti formali

16 CIL XIV, 246: Imp(eratore) Caesare T(ito) Aelio Hadriano Antonino Aug(usto) / Pio, p(atri) p(atriae), III, M(arco) Aelio Aurelio Caesare co(n)s(ulibus). I Ordo corporator(um) qui pecuniam ad ampliand(um) templum contuler(unt). // Patroni: / T(itus) Prifernius Sex(ti) fil(ius) / Paetus Rosianus / Geminus, / M(arcus) Stlaccius Albinus / Trebellius Sallustius / Rufus, / M(arcus) Sedatius C(ai) fil(ius) / Severianus, / T(itus) Prifernius T(iti) f(ilius) Paetus / Rosianus Geminus f(ilius), / M(arcus) Sedatius M(arci) fil(ius) / Severus Iulius Reginus, / C(aius) Allius C(ai) f(ilius) / Fuscianus, / T(itus) Statilius 
per appartenere a un collegium, tuttavia lo scopo esplicitato nell'intestazione sembrerebbe far crollare uno dei quattro elementi caratterizzanti di un'associazione postulati da Francesco De Robertis: «la permanenza dello scopo, importante la concettuale continuità nel tempo, sia pure solo nella intenzione dei componenti» e ancora "gli aderenti cioè non si devono proporre uno scopo passeggiero, come avviene nelle riunioni»[De Robertis 1971a, 6]. Infatti, nel momento in cui si fosse raggiunta la somma necessaria ad ampliandum templum, tali associazioni avrebbero cessato di esistere, e dunque già in origine tutti gli ascritti a

T(iti) f(ilius) / Taurus, / Ti(berius) Aterius / Saturninus, / C(aius) Pantuleius / Graptiacus, / C(aius) Allius C(ai) f(ilius) / Fuscus. // Quinq(uennalis) perpet(uus): C(aius) Sossius Benedictus; / quinquenn(alis): / A(ulus) Egrilius Augustal(is); I quinquennalic(ii): / P(ublius) Sulpicius Hera, / L(ucius) Naevius Saecular(is). / Quintiliis co(n)s(ulibus), / quinquenn(alis): / A(ulus) Egrilius Faustus. / Silvano et Augurin(o) / co(n)s(ulibus), q(uin)q(uennales): / T(itus) Fisevius Nicephor(us), / L(ucius) Naevius Proculus. I Laeliano et Pastore / co(n)s(ulibus), q(uin)q(uennalis): / T(itus) Fisevius Priscianus. I Claro et Cethego co(n)s(ulibus), I q(uin)q(uennalis): / M(arcus) Magius Marsus. / Maximo et Orfito co(n)s(ulibus), I q(uin)q(uennales): / L(ucius) Helvius Restitutus; / A(ulus) Egrilius Faustus, / testamento reli/qu(i)t HS IIII m(ilia) n(ummum), sub / ea condicione uti / ex usuris s(ummae) s(uprae) s(criptae) V Kal(endas) / Dec(embras) omnibus annis / epulentur. Seguono su cinque colonne i nomi di circa 181 membri della plebs. CIL XIV, 5356 (fr. a, b, c) + CIL XIV, 5373 (fr. d) = Cicerchia, Marinucci 1992, pp. 188-190, nr. C46 = EDR 110171 (Raffaella Marchesini): [Imp(eratore) [[Com]modo [I]I et]] P(ublio) Martio Ve] ro II co(n)s(ulibus). I [O]rdo corpor(atum) qui pec[u]nia ad t [empl(um) contu]lerunt. // Patroni c(larissimi) v(iri): / L(ucius) Saenius / Donatus / Saturninus. // Equit(es) Rom(ani): / L(ucius) Manlius Priscus. // Quin[q(uennales)]: / L(ucius) Aeli[u] s / Trophimus ,/ L(ucius) Calpurnius / Cirratus p(er)p(etuus). / M(arco) Petronio Mamertino, / Q(uinto) Tineio Rufo co(n)s(ulibus), / L(ucius) Aelius Probus / quinq(uennalis), / [[------]]. / L(ucio) Bruttio Crispin//o, / L(ucio) Roscio Aeliano / co(n)s(ulibus), // L(ucius) Aelius Marcia/nus quinq(uennalis) p(er)p(etuus), / L(ucius) Valerius / Callistianus / q(uin)q(uennalis). CIL XIV, 5374 = Cicerchia, Marinucci 1992, pp. 198-200, nr. C60 = EDR 110209 (Raffaella Marchesini): ------ / [Ordo corporator(um) ---] / qui ad ampliand(um) templum contuler(unt)]. // Patroni: / Cn(aeus) Sentius Felix, / L(ucius) Munatius / Cascellianu[s], / Cn(aeus) Sentiu[s] / Clod[ianus]. / Quinquen[nalis] / perpetu[us]: / L(ucius) Laberius Fau[stus]. Quinquennaliç[ii]: / P(ublius) Saenius Pa+[--], / L(ucius) Laberius Fau[stus], / ----- / [C(aius) Sossius B] enedictus, / [Pontiano e] t Atiliâno co(n)s(ulibus), / [quinquen]nales: / [P(ublius) Sulpicius] Hera / [L(ucius) Naevius S]ạeclaris. // Seguono su più di due colonne un numero imprecisato di membri appartenenti alla plebs. 
questi albi dovevano essere consapevoli della non continuità nel tempo, nemmeno potenziale, dello scopo per cui si riunivano.

Tuttavia, grazie alle analisi compiute da Antonio Licordari, Halsey Royden e Nicolas Tran su personaggi noti degli alba CIL XIV, 246 e 5374', principalmente patroni e quinquennales, è stato possibile identificare l'ordo corporatorum qui pecuniam ad ampliandum templum contulerunt con il corpus lenunculariorum traiectus Luculli [Licordari 1987, 158; Royden 1988, 33-34; Tran 2012, 327-329].

Rimangono solo da capire le circostanze nelle quali i lenuncularii traiectus Luculli scelsero di presentarsi come i corporati qui pecuniam ad ampliandum templum contulerunt. Nicolas Tran, procedendo per analogia con un altro caso molto simile, giunge a una spiegazione abbastanza soddisfacente. Egli prende in considerazione un'iscrizione, dedicata al numen della Domus Augusta, realizzata per eternare la memoria dei lavori compiuti dai lenuncularii traiectus Luculli, presso la riva del Tevere. Da quest'iscrizione sappiamo che la corporazione ricevette l'autorizzazione direttamente da Ti. Iulius Ferox, curator alvei Tiberis et riparum tra il 101 e il 103 d.C., tuttavia in questa epigrafe non vi è alcuna menzione della costruzione compiuta. Tale assenza si può spiegare attraverso il contesto, ovvero l'epigrafe non necessitava di rendere esplicite quali costruzioni fossero state eseguite poiché «ses lecteurs l'avaient devant les yeux» [Tran 2012, 330]. Dunque, per analogia, i lenuncularii traiectus Luculli non avevano sentito il bisogno di specificare il loro essere lenuncularii su questi alba, poiché "celles-ci étaient affichées au milieu d'un décor constitué d'inscriptions religieuses ou impériales ne laissant aucun doute sur l'occupation du templum par les lenuncularii traiectus Luculli» [Tran 2012, 330].

Infine il corpus (scaphariorum) traiectus Rusticeli era un'associazione preposta a traghettare merci e persone da una sponda all'altra del Tevere,

Per quanto riguarda invece CIL XIV $5356+5373$ probabilmente siamo davanti a un altro collegium a noi ignoto. Per approfondimenti si veda [Ciambelli 2016, 82-84] 
così come facevano i lenuncularii traiectus Luculli. Qui l'unica differenza è il mezzo di trasporto che non è un lenunculus, ma una scapha, una diversa specie di imbarcazione utilizzata principalmente nel mondo romano come scialuppa a rimorchio delle grandi navi quando erano in mare aperto, mentre durante le manovre d'attracco erano queste piccole barche che guidavano le grandi imbarcazioni [Le Gall 1952, 220-222; Rougé 1966, 192-193; De Salvo 1992, 166-168] ${ }^{18}$. Il nome di questa corporazione è dovuto alla posizione del loro attracco presso i praedia Rusticeliana, possedimenti di proprietà del fisco imperiale così denominati dal nome dai precedenti proprietari, i Rusticeli [CébeillacGervasoni, Caldelli, Zevi 2010, 194].

Riassumendo, i collegia che annoverano senatori nel ruolo di patroni erano tutti legati all'attività annonaria, o comunque inseriti all'interno del settore dei trasporti connesso all'ambiente portuale, ed erano perciò di più ampio respiro rispetto alle semplici corporazioni utili alla sola comunità cittadina.

È bene notare che tutti i personaggi di ordine senatorio, per i quali è stato possibile ricostruire la carriera, raggiunsero il consolato e alcuni svolsero anche un ruolo di primo piano nella grande politica imperiale. Ad esempio, L. Fabius Cilo Septiminus Catinius Acilianus Lepidus Fulcinianus (nr. 3) ${ }^{19}$ fu uno dei marescialli di Settimio Severo durante le guerre civili seguite alla scomparsa di Pertinace [Ciambelli 2016, 137142], oppure C. Allius Fuscus (nr. 2) fu ucciso per ordine di Commodo poiché invischiato in una congiura di palazzo [Ciambelli 2016, 111113]. Dunque a Ostia in veste di patroni figuravano anche personaggi di spicco dell'élite imperiale.

Guido Clemente, nel suo fondamentale studio sul patronato dei collegia, non riconosceva un ruolo attivo per il patrono senatore, considerato del tutto avulso alla comunità e alle logiche collegiali, anzi riteneva la coop-

18 Non., XIII, 858 (Lindsay): Scaphae sunt naviculae quae maiores naves consecuntur.

19 I numeri fra parentesi si riferiscono alla tabella 1 inserita in coda al testo. 
tatio patroni nei confronti dei patres conscripti solamente un mezzo per i collegiati di accrescere il prestigio dell'associazione stessa. Così, infatti, scriveva lo studioso:

I patroni dell'ordine senatorio che abbiamo elencato rappresentano, in generale, un ceto estraneo agli interessi della città e delle associazioni. Non sono note infatti connessioni fra le attività dei collegi e i patroni senatori da questi cooptati, anche quando i personaggi in questione appartengono alla nobiltà locale. Il grande sviluppo delle associazioni nel II secolo ha evidentemente consentito ad esse di assicurarsi il patronato di uomini di rilievo dell'aristocrazia locale o romana, estranei all'ambiente del collegio, e scelti per ragioni di prestigio, secondo il più ovvio rapporto di patronato: per questo, anche i tipi di collegio patrocinati da senatori sono di natura assai diversa [Clemente 1972, 203].

Questa chiave interpretativa negherebbe tuttavia l'esistenza di un rapporto asimmetrico, fondato sullo scambio di beni e servizi, e dunque il fondamento stesso del patronato. I patroni senatori erano sì del tutto estranei al contesto urbano ostiense e certamente non necessitavano di onori da parte dei collegi locali per accrescere il loro prestigio e veder riconosciuto il loro ruolo nella società romana. Infatti i supporti su cui sono ricordati sono esclusivamente alba collegiali ${ }^{20}$, datati circa alla seconda metà del II sec. d.C., mentre sono totalmente assenti basi di statue o epigrafi onorarie. Ritengo però probabile che, così come il notabile municipale riceveva come contropartita delle sue elargizioni onori da parte dei collegia che ne aumentavano la visibilità, nonché il prestigio sociale per riconfermare il suo ruolo all'interno del tessuto cittadino, così il senatore patrono dovesse avere un tornaconto di altro tipo. A mio avviso l'intervento di personaggi di tale calibro presso il mondo corporativo, sorto attorno ai porti imperiali di Roma, fu motivato da interessi economici e commerciali: un senatore accettava la cooptatio patroni da parte di un'associazione professionale per intessere, attraverso

\footnotetext{
${ }^{20}$ CIL XIV, 246; 247; 250; 251. AE 2001, 622.
} 
il più aristocratico dei legami, rapporti con il mondo professionale e associativo utili ai traffici commerciali e all'accrescimento del proprio potere economico. Non è da dimenticare, infatti, che se tutte le strade portavano a Roma, quasi tutte le rotte marine conducevano a Ostia.

Ora, beninteso, ai senatori e ai loro figli era stato proibito, attraverso il plebiscitum Claudianum del 219/218 a.C., di possedere navi sopra le 300 anfore di capacità, provvedimento che li escludeva dalla pratica diretta del commercio marittimo ${ }^{21}$. Da un passo del giurista Scevola, raccolto nel Digesto, apprendiamo che tale disposizione, riguardante qui invero il possesso delle navi in generale senza la specificazione del tonnellaggio, era confluita all'interno della Lex Iulia de repetundis, proposta da Giulio Cesare durante il consolato del 59 a.C. e ancora in vigore durante il principato di Marco Aurelio, epoca in cui visse il giurista ${ }^{22}$. Inoltre, grazie alla scoperta nel 1955 di un frammento delle Sententiae paoline, nel quale è ribadito il divieto dei senatori di lucrare attraverso il commercio marittimo, possiamo avere la prova che la normativa presente nel plebiscitum Claudianum era ancora in vigore durante il periodo di attività di Paolo, ovvero il principato di Alessandro Severo. Nondime-

${ }_{21}$ Il plebiscitum Claudianum del 219/218 a.C., a volte ricordato impropriamente come lex Claudia, è menzionato nelle fonti letterarie per primo da Tito Livio [Liv., XXI, 63, 3-4]: invisus etiam patribus ob novam legem, quam Q. Claudius tribunus plebis adversus senatum atque uno patrum adiuvante C. Flaminio tulerat, ne quis senator cuive senator pater fuisset maritimam navem, quae plus quam trecentarum amphorarum esset, haberet. Id satis habitum ad fructus ex agris vectandos; quaestus omnis patribus indecorus visus. Per Livio il limite di trecento anfore era sufficiente per il trasporto dei prodotti agricoli, mentre qualsiasi altra speculazione era da ritenere indecorosa per i senatori. Il dibattito sul plebiscitum Claudianum interno alla moderna storiografia ha prodotto una notevole mole di studi in merito. Per l'elenco degli studi principali e per una presentazione sintetica delle posizioni assunte dai vari studiosi, si veda da ultimo Marianne Coudry [Coudry 2007].

${ }_{22}$ Dig., L, 5, 3 (Scevola): Libro tertio regularum. His, qui naves marinas fabricaverunt et ad annonam populi Romani praefuerint non minores quinquaginta milium modiorum aut plures singulas non minores decem milium modiorum, donec hae naves navigant aut aliae in earum locum, muneris publici vacatio praestatur ob navem. Senatores autem hanc vacationem habere non possunt, quod nec habere illis navem ex lege Iulia repetundarum licet. 
no, in questo passo il giurista severiano specifica che ai patres conscripti era proibito navem in quaestum habere, ovvero il possesso di una qualsiasi nave da adibire a trasporti esercitati a fine di lucro ${ }^{23}$.

Come si è potuto notare, il divieto riguardava solamente il possesso di navi da parte dei patres conscripti e dei loro figli, «i senatori, cioè, non potevano farsi armatori in prima persona» [Clemente 1983, 255], ma nulla avrebbe proibito loro di esercitare le attività commerciali [Guarino 1982; Clemente 1983; Tchernia 2007]. Per far ciò, e aggirare così il divieto imposto per legge, dovevano usufruire dell'appoggio del ceto medio mercantile. L'interessamento dei patres conscripti per le associazioni ostiensi potrebbe essere visto in quest'ottica: non sembra essere un caso infatti che i senatori a Ostia siano registrati come patroni esclusivamente in alba appartenenti a collegia di battellieri attivi presso i porti imperiali.

Per alcuni di questi personaggi analizzati è stato possibile, soprattutto attraverso l'incrocio di informazioni forniteci dalla documentazione archeologica, ricostruire un'immagine più o meno fedele delle produzioni dei loro latifondi. L. Fabius Cilo (nr. 3), nativo della Betica, aveva in questa regione vasti possedimenti legati alla produzione di olio, destinato soprattutto a foraggiare le truppe romane lungo il limes germanico $^{24}$. Egli, in base ai ritrovamenti dei sigilli impressi sulle anfore di tipo Dressel 20, può essere considerato uno dei primi produttori ed esportatori di olio dell'epoca severiana (figura 4).

La famiglia dei Prifernii Paeti, presente negli alba ostiensi con ben tre dei suoi esponenti (T. Prifernius Paetus Rosianus Geminus Laecanius Bas-

23 Paolo, Sent., (Leid.), III, 1, 4-7: Senatores parentesue eorum in quorum potestate sunt vectigalia publica conducere, navem in quaestum habere equosue curules praebendos suscipere prohibentur: idque factum repetundarum lege vindicatur. Per l'edizione di questo frammento leidense delle Sententiae paoline utile è lo studio di Feliciano Serrao [Serrao 1956, 19-23].

24 Sulle anfore con il sigillo di Cilone si vedano Remesal Rodríguez 1989 [143145] e Chic García 1994. Per un approfondimento sull'olio della Betica Remesal Rodríguez 1998. 


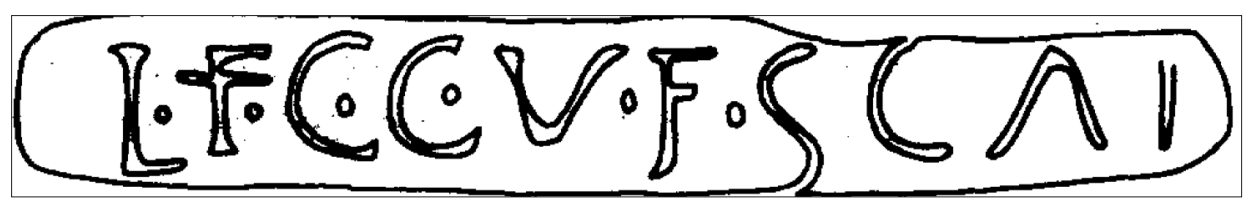

Figura 4. Sigillo di Cilone rinvenuto su un anfora di tipo Dressel 20 in Germania. La sigla cosi può essere sciolta: L(ucius) F(abius) C(ilo) C(larissimus) V(ir) F(iglina) SCAL(ensia). [Remesal Rodríguez 1989, 143].

sus Fronto nr. 6, T. Prifernius Paetus Rosianus Geminus nr. 7 e T. Prifernius Paetus Rosianus Nonius Agricola C. Labeo Testius Geminus nr. 8), era una delle maggiori produttrici d'olio dell'Istria [Granino Cecere 2003, 21; Zaccaria 2014, 304]. La presenza di questa gens per due generazioni (figura 5), tra il secondo quarto e la fine del II sec. d.C., tra le fila dei patroni dei lenuncularii tabularii, dei lenuncularii traiectus Luculli e degli scapharii traiectus Rusticeli sembra essere l'esempio più evidente della volontà di proteggere interessi famigliari, di natura commerciale ed economica, presso le associazioni al servizio dei porti imperiali di Ostia. Infine, anche la famiglia dei Sedatii, originaria di Limonum, fu presente per due generazioni (M. Sedatius Severianus Iulius Acer Metilius Nepos Rufinus Ti. Rutilianus Censor nr. 11 e M. Sedatius Severus Iulius Reginus

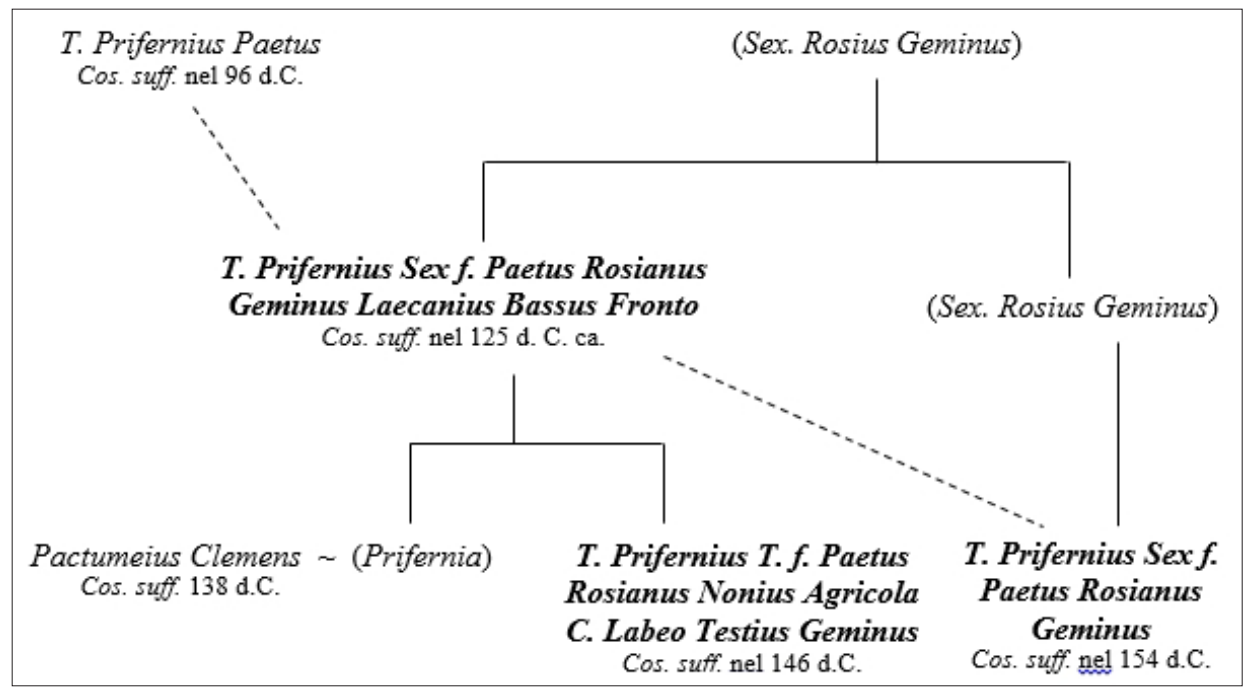

Figura 5. Stemma dei Prifernii Paeti. Con la linea continua si indica la discendenza naturale, mentre con la linea tratteggiata l'adozione. Sono inclusi tra parentesi i personaggi ignoti, ma dei quali è possibile ipotizzare parte del nome. 
nr. 12), durante la seconda metà del II sec. d.C., tra i patroni dei lenuncularii tabularii, dei lenuncularii traiectus Luculli e degli scapharii traiectus Rusticeli. Di questa gens conosciamo i forti interessi economici sia lungo la valle della Loira sia in terreni sparsi nei dintorni di Roma [Picard 1981; Tran 2012].

Per via della lacunosità delle fonti non è possibile individuare dove si concentrassero i possedimenti degli altri patroni senatori e attraverso quali mezzi mantenessero la loro ricchezza e quindi il loro status all'interno della società romana, ma si può ipotizzare che anche loro abbiano voluto intessere una relazione di patronato con le suddette associazioni per poter difendere al meglio i loro interessi commerciali.

Un altro motivo che potrebbe aver spinto un numero così consistente di senatori a interessarsi del mondo associativo ostiense può essere intravisto nella disposizione di Traiano circa l'obbligo del possesso di terre in Italia per i senatori. Da una epistula di Plinio il Giovane, indirizzata all'amico Nepote, apprendiamo che l'imperatore Traiano impose ai patres conscripti di possedere almeno un terzo dei loro terreni in Italia, ciò per legare anche il sempre più consistente numero di senatori di origine provinciale al territorio italiano ${ }^{25}$. Nella stessa missiva vi è scritto che i possedimenti più richiesti e ambiti erano quelli situati nel suburbium di Roma, poiché logicamente i provinciali ricercavano poderi il

25 Plin., Ep., VI, 19: C. PLINIVS NEPOTI SVO S. Scis tu accessisse pretium agris, praecipue suburbanis? Causa subitae caritatis res multis agitata sermonibus. Proximis comitiis honestissimas voces senatus expressit: 'Candidati ne conviventur, ne mittant munera, ne pecunias deponant.' Ex quibus duo priora tam aperte quam immodice fiebant; hoc tertium, quamquam occultaretur, pro comperto habebatur. Homullus deinde noster vigilanter usus hoc consensu senatus sententiae loco postulauit, ut consules desiderium universorum notum principi facerent, peterentque sicut aliis vitiis huic quoque prouidentia sua occurreret. Occurrit; nam sumptus candidatorum, foedos illos et infames, ambitus lege restrinxit; eosdem patrimonii tertiam partem conferre iussit in ea quae solo continerentur, deforme arbitratus (et erat) honorem petituros urbem Italiamque non pro patria sed pro hospitio aut stabulo quasi peregrinantes habere. Concursant ergo candidati; certatim quidquid venale audiunt emptitant, quoque sint plura venalia efficiunt. Proinde si paenitet te Italicorum praediorum, hoc vendendi tempus tam hercule quam in provinciis comparandi, dum idem candidati illic vendunt ut hic emant. Vale. Per un commento a tale lettera [Sherwin-White 1966, 376-378]. 
più possibile vicini alla capitale dove dovevano obbligatoriamente recarsi per svolgere le loro attività.

$\grave{E}$ dunque possibile ipotizzare, in base alle conoscenze di cui disponiamo, che i senatori attestati come patroni a Ostia, per la maggior parte di origine provinciale, avessero dei possedimenti nei dintorni dell'Urbe. In tal caso il porto di Ostia avrebbe assunto il ruolo di trait d'union tra $\mathrm{i}$ possedimenti italiani e quelli provinciali, o più semplicemente sarebbe stato più facile per tali senatori entrare in contatto con la comunità ostiense data la prossimità dei loro terreni ${ }^{26}$.

\section{Conclusioni}

Attraverso l'indagine del fenomeno del patronato immerso nel particolare contesto ostiense è possibile notare, in base alla documentazione a nostra disposizione, un interesse dei senatori presso le associazioni professionali inserite nel circuito dei porti imperiali. Infatti, anche i patres conscripti dovevano, per il consolidamento della loro base economica, essenziale per la loro stabilità sociale ${ }^{27}$, intessere rapporti di dipendenza costanti con la plebs media, la quale presso Ostia era per la maggior parte imbrigliata all'interno di schemi collegiali. Tali rapporti di dipendenza in questa colonia si esplicitavano anche attraverso l'instaurarsi di relazioni di patronato che dunque rendono tangibile questo fenomeno.

\footnotetext{
26 Questa teoria basata sulla maggior vicinanza e dunque reperibilità dei senatori viene presentata in [Tran 2012, 343-344] a proposito di M. Sedatius Severianus Iulius Acer Metilius Nepos Rufinus Ti. Rutilianus Censor (nr. 11), ma può a mio avviso essere estesa anche agli altri patres conscripti patroni di associazioni professionali ad Ostia.

27 È bene ricordare a questo proposito che Augusto aveva fissato per i senatori un censo minimo di un milione di sesterzi, Svet., Aug., 41, 1: senatorum censum ampliavit ac pro octingentorum milium summa duodecies sestertium taxavit supplevitque non habentibus.
} 


\section{Appendice: i patroni delle associazioni professionali di Ostia di estrazione senatoria}
Patrono
Datazione Patronato
Riferimenti
del patro-
prosopografici

nato

1 C. Allius Metà del II CIL XIV, 250: Lenuncularii ta- PIR $^{2}, \mathrm{~A}, 544$

Fuscianus sec.d.C. bularii

CIL XIV, 246: Ordo corpora-

torum qui pecunia ad templum

contulerunt (lenuncularii traiec-

tus Luculli)

2 C. Allius Metà del II CIL XIV, 251: Lenuncularii ta- PIR², A, 545

Fuscus sec. d.C. bularii

CIL XIV, 246: Ordo corpora-

torum qui pecunia ad templum

contulerunt (lenuncularii traiec-

tus Luculli)

3 L. Fabius Fine II sec. CIL XIV, 251: Lenuncularii ta- $\mathrm{PIR}^{2}$, F, 27

Cilo Septi- d.C. inizio bularii

minus Cati- III sec. d.C.

nius Acilia-

nus Lepidus

Fulcinianus

4 T. Haterius Metà del II CIL XIV, 246: Ordo corpora- PIR ${ }^{2}, \mathrm{H}, 32$

Saturninus sec.d.C. torum qui pecunia ad templum

contulerunt (lenuncularii traiec-

tus Luculli)

5 C. Pantule- Metà del II CIL XIV, 246: Ordo corpora- PIR², P, 96 ius Graptia- sec. d.C. torum qui pecunia ad templum cus contulerunt (lenuncularii traiectus Luculli) 


\begin{tabular}{|c|c|c|c|c|}
\hline & Patrono & $\begin{array}{l}\text { Datazione } \\
\text { del patro- } \\
\text { nato }\end{array}$ & Patronato & $\begin{array}{l}\text { R iferimenti } \\
\text { prosopografici }\end{array}$ \\
\hline 6 & $\begin{array}{l}\text { T. Prifer- } \\
\text { nius Paetus } \\
\text { Rosianus } \\
\text { Geminus } \\
\text { Laecanius } \\
\text { BassusFronto }\end{array}$ & $\begin{array}{l}\text { Prima metà } \\
\text { del II sec. } \\
\text { d.C. }\end{array}$ & $\begin{array}{l}\text { CIL XIV, 246: Ordo corpora- } \\
\text { torum qui pecunia ad templum } \\
\text { contulerunt (lenuncularii traiec- } \\
\text { tus Luculli) }\end{array}$ & $\mathrm{PIR}^{2}, \mathrm{P}, 938$ \\
\hline 7 & $\begin{array}{l}\text { T. Prifernius } \\
\text { Paetus Ro- } \\
\text { sianus Ge- } \\
\text { minus }\end{array}$ & $\begin{array}{l}\text { Metà del II } \\
\text { sec. d.C. }\end{array}$ & $\begin{array}{l}\text { CIL XIV, 250: Lenuncularii ta- } \\
\text { bularii }\end{array}$ & $\mathrm{PIR}^{2}, \mathrm{P}, 937$ \\
\hline 8 & $\begin{array}{l}\text { T. Prifernius } \\
\text { Paetus Ro- } \\
\text { sianus No- } \\
\text { nius Agrico- } \\
\text { la C. Labeo } \\
\text { Testius Ge- } \\
\text { minus }\end{array}$ & $\begin{array}{l}\text { Metà del II } \\
\text { sec. d.C. }\end{array}$ & $\begin{array}{l}\text { CIL XIV, 247: Scapharii traiec- } \\
\text { tus Rusticeli } \\
\text { CIL XIV, 246: Ordo corpora- } \\
\text { torum qui pecunia ad templum } \\
\text { contulerunt (lenuncularii traiec- } \\
\text { tus Luculli) }\end{array}$ & $\mathrm{PIR}^{2}, \mathrm{P}, 939$ \\
\hline 9 & $\begin{array}{l}\text { L. Rufius } \\
\text { Clarus Gal- } \\
\text { lus? }\end{array}$ & $\begin{array}{l}\text { Metà del II } \\
\text { sec. d.C. }\end{array}$ & $\begin{array}{l}\text { CIL XIV, 248: Collegio igno- } \\
\text { to }\end{array}$ & $\mathrm{PIR}^{2}, \mathrm{R}, 154$ \\
\hline 10 & $\begin{array}{l}\text { L. Saenius } \\
\text { Donatus } \\
\text { Saturninus }\end{array}$ & $\begin{array}{l}\text { S e c o n d a } \\
\text { metà del II } \\
\text { sec. d.C. }\end{array}$ & $\begin{array}{l}\text { CIL XIV, } 5356+5373 \text { : Ordo } \\
\text { corporatorum qui pecunia ad } \\
\text { templum contulerunt (collegio } \\
\text { ignoto) }\end{array}$ & $\mathrm{PIR}^{2}, \mathrm{~S}, 58$ \\
\hline
\end{tabular}




\begin{tabular}{|c|c|c|c|c|}
\hline & Patrono & $\begin{array}{l}\text { Datazione } \\
\text { del patro- } \\
\text { nato }\end{array}$ & Patronato & $\begin{array}{l}\text { R iferimenti } \\
\text { prosopografici }\end{array}$ \\
\hline 11 & $\begin{array}{l}\text { Sedatius } \\
\text { Severia- } \\
\text { nus Iulius } \\
\text { Acer Meti- } \\
\text { lius Nepos } \\
\text { Rufinus Ti. } \\
\text { Rutilianus } \\
\text { Censor }\end{array}$ & $\begin{array}{l}\text { Metà del II } \\
\text { sec. d.C. }\end{array}$ & $\begin{array}{l}\text { CIL XIV, 246: Ordo corpora- } \\
\text { torum qui pecunia ad templum } \\
\text { contulerunt (lenuncularii traiec- } \\
\text { tus Luculli) } \\
\text { CIL XIV, 247: Scapharii traiec- } \\
\text { tus Rusticeli } \\
\text { CIL XIV, 248: Collegio igno- } \\
\text { to } \\
\text { CIL XIV, 250: Lenuncularii ta- } \\
\text { bularii }\end{array}$ & $\mathrm{PIR}^{2}, \mathrm{~S}, 306$ \\
\hline 12 & $\begin{array}{l}\text { M. Sedatius } \\
\text { Severus Iu- } \\
\text { lius Reginus }\end{array}$ & $\begin{array}{l}\text { Metà del II } \\
\text { sec. d.C. }\end{array}$ & $\begin{array}{l}\text { CIL XIV, 246: Ordo corpora- } \\
\text { torum qui pecunia ad templum } \\
\text { contulerunt (lenuncularii traiec- } \\
\text { tus Luculli) } \\
\text { CIL XIV, 247: Scapharii traiec- } \\
\text { tus Rusticeli } \\
\text { CIL XIV, 248: Collegio igno- } \\
\text { to } \\
\text { CIL XIV, 250: Lenuncularii ta- } \\
\text { bularii }\end{array}$ & $\mathrm{PIR}^{2}, \mathrm{~S}, 307$ \\
\hline 13 & $\begin{array}{l}\text { T. Statilius } \\
\text { Taurus }\end{array}$ & $\begin{array}{l}\text { Metà del II } \\
\text { sec. d.C. }\end{array}$ & $\begin{array}{l}\text { CIL XIV, 246: Ordo corpora- } \\
\text { torum qui pecunia ad templum } \\
\text { contulerunt (lenuncularii traiec- } \\
\text { tus Luculli) }\end{array}$ & $\mathrm{PIR}^{2}, \mathrm{~S}, 857$ \\
\hline 14 & $\begin{array}{l}\text { M. Stlac- } \\
\text { cius Albinus } \\
\text { Trebellius } \\
\text { Sallustius } \\
\text { Rufus }\end{array}$ & $\begin{array}{l}\text { Metà del II } \\
\text { sec. d.C. }\end{array}$ & $\begin{array}{l}\text { CIL XIV, 246: Ordo corpora- } \\
\text { torum qui pecunia ad templum } \\
\text { contulerunt (lenuncularii traiec- } \\
\text { tus Luculli) }\end{array}$ & $\mathrm{PIR}^{2}, \mathrm{~S}, 919$ \\
\hline
\end{tabular}




$\begin{array}{lll}\text { Patrono } & \begin{array}{l}\text { Datazione Patronato } \\ \text { del patro- } \\ \text { nato }\end{array} & \begin{array}{l}\text { Riferimenti } \\ \text { prosopografici }\end{array} \\ 15 \text { M. Umbi- } & \text { Fine del II CIL XIV 251: Lenuncularii ta- } \text { PIR }^{1}, \mathrm{U}, 589 \\ \text { lius Maxi- } & \text { sec. d.C. ini- bularii } \\ \text { minus } & \text { zi del III sec. AE 2001, 622: Collegio ignoto } & \\ & \text { d.C. }\end{array}$

Tabella 1. I patroni delle associazioni professionali di Ostia di estrazione senatoria.

\section{Indice delle abbreviazioni}

$\mathrm{AE}=$ Année Epigraphique, $1888-$.

CIL = Corpus Inscriptionum Latinarum, 1847-.

EDR $=$ Epigraphic Database Roma, <www.edr-edr.it $>$

ILS = H. Dessau, Inscriptiones latinae selectae, Berlin, 1892-1916.

LSA = Last Statues of Antiquity, <http://laststatues.classics.ox.ac.uk>

OCRE $=$ Online Coins of the Roman Empire, <www.numismatics.org/ocre >

PIR = Prosopographia Imperii Romani saec. I. II. III., Editio altera, Berlin, 1933-.

PLRE = The Prosopography of the Later Roman Empire.

$\mathrm{RIC}=$ Roman Imperial Coinage.

\section{Bibliografia}

Bianchi Bandinelli R. 1969, Roma. L'arte romana nel centro del potere, Milano: Rizzoli.

Casson L. 1965, Harbour and river boats of ancient Rome, "The Journal of Roman studies», 55: 31-39.

Cébeillac-Gervasoni M., Caldelli M. L., Zevi F. 2010, Epigrafia latina. Ostia: cento iscrizioni in contesto, Roma: Quasar.

Chevallier R. 1986, Ostie antique, ville \& port, Paris: Les Belles Lettres.

Chic García G. 1994, Los centros productores de las ánforas de L. F. C., "Hispania antiqua: revista de historia antigua», 18: 171-233. 
Ciambelli S. 2016, Ob merita eius. I patroni delle associazioni professionali di Ostia, Tesi di Laurea, Alma Mater Studiorum - Università di Bologna.

Cicerchia P., Marinucci A. 1992, Scavi di Ostia XI. Le Terme del Foro o di Gavio Massimo, Roma: Istituto Poligrafico e Zecca dello Stato.

Clemente G. 1972, Il patronato nei collegia dell'Impero Romano, "Studi Classici e Orientali», 21: 142-229.

Clemente G. 1983, Il plebiscito Claudio e le classi dirigenti romane nell'età dell'imperialismo, "Ktèma», 8: 253-259.

Coudry M. 2007, Loi Claudia interdisant aux sénateurs la possession d'un navire de plus de 300 amphores, «Lepor. Leges Populi Romani», http://www.cn-telma.fr/lepor/notice96/.

Cristofori A. 2004, Non arma virumque. Le occupazioni nell'epigrafia del Piceno, Bologna: Lo scarabeo.

De Robertis F. 1971, Storia delle corporazioni e del regime associativo nel mondo romano, vol. I, Bari: Adriatica.

De Salvo L. 1992, Economia privata e pubblici servizi nell'impero romano. I corpora naviculariorum, Messina: Sampieri.

Fustel De Coulanges D. N. 1900, Histoire des institutions politiques de l'ancienne France. Les origines du système féodal. Le bénéfice et le patronat pendant l'époque Mérovingienne (II ed.), Paris.

Gelzer M. 1962, Die Nobilität der römischen Republik, «Kleine Schriften», vol. I, Wiesbaden: F. Steiner, 17-135.

Granino Cecere M. G. 2003, La carriera di T. Prifernius Paetus Rosianus Geminus in un'iscrizione onoraria di Trebula Mutuesca, in Angeli Bertinelli M. G., Donati A. (eds.) 2003, Serta Antiqua et Mediaevalia VI. Usi e abusi epigrafici, atti del Colloquio internazionale di Epigrafia Latina (Genova 20-22 settembre 2001) Roma: Bretschneider, 1-28.

Guarino A. 1982, Quaestus omnis patribus indecorus, «Labeo», 28: 7-16.

Le Gall J. 1953, Le Tibre fleuve de Rome dans l'antiquité, Paris: Presses Universitaires de France.

Licordari A. 1987, I «lenuncularii traiectus Luculli» ad Ostia, «Miscellanea greca e romana», 12: 149-161.

Meiggs R. 1973, Roman Ostia, Oxford: Clarendon Press.

Minervini G. 1852, Nuove osservazioni intorno la voce Decatrenses, la quale s'incontra in alcune iscrizioni puteolane, "Memorie della regale Accademia Ercolanese di Archeologia», Napoli: 349-364.

Pavolini C. 1986, La vita quotidiana a Ostia, Roma-Bari: Laterza. 
Remesal Rodríguez J. 1989, Tres nuevos centros productores de anforas Dressel 20 y 23 los sellos de Lucivs Fabivs Cilo, "Ariadna", 6: 120-153.

Remesal Rodríguez J. 1998, Betican olive oil and the Roman economy, "JRA suppl.», 29: 183-199.

Picard G. C. 1981, Ostie et la Gaule de l'Ouest, "Mélanges de l'École française de Rome - Antiquité», 93 (2): 883-915.

Rougé J. 1966, Recherches sur l'organisation du commerce maritime en Méditerranée sous l'Empire romain, Paris: S.E.V.P.E.N.

Royden H. 1988, The Magistrates of the Roman Professional Collegia in Italy from the First to the Third Century A.D., Pisa: Giardini Editori e Stampatori.

Saller R. 1982, Personal patronage under the early Empire, Cambridge: Cambridge University Press.

Serrao F. 1956, Il frammento leidense di Paolo, problemi di diritto criminale romano, Roma: Giuffrè.

Sherwin-White A. N. 1966, The letters of Pliny, a historical and social commentary, Oxford: Clarendon Press.

Sirks B. 1991, Food for Rome. The legal structure of the transportation and processing of supplies for the imperial distributions in Rome and Constantinople, Amsterdam: Gieben.

Tchernia A. 2007, Le plebiscitum Claudianum, «Vocabulaire et expression de l'économie dans le monde antique», Pessac: Ausonius Éditions, 253-278.

Tran N. 2012, Un Picton à Ostie: M. Sedatius Severianus et les corps de Lénunculaires sous le principat d'Antonin le Pieux, «Revue des études anciennes», 114 (2): 323-344.

Tran N. 2014, C. Veturius Testius Amandus, les cinq corps de lénunculaires d'Ostie et la batellerie tibérine au début du IIle siècle, "Mélanges de l'École française de Rome Antiquité», 126 (1), http://mefra.revues.org/2043.

Waltzing J.-P. 1895, Étude historique sur les corporations professionnelles chez les Romains, vol. I, Louvain: Peeters.

Waltzing J.-P. 1896, Étude historique sur les corporations professionnelles chez les Romains, vol. II, Louvain: Peeters.

Waltzing J.-P. 1899, Étude historique sur les corporations professionnelles chez les Romains, vol. III, Louvain: Peeters.

Waltzing J.-P. 1900, Étude historique sur les corporations professionnelles chez les Romains, vol. IV, Louvain: Peeters.

Zaccaria C. 2014, A proposito di un nuovo senatore de Nesactium (regio X): T. Prifernius Paetus C. Fil. Settidianus Firmus, cos. suff. e legato della Moesia superior in età traianea, in Caldelli M. L., Gregori G. L. (eds.) 2014, Epigrafia e ordine senatorio, 30 anni dopo, Roma: Quasar, 299-312.

Zevi F. 2001, Histoire et topographie des ports de Rome, «Pallas», 55: 267-284. 